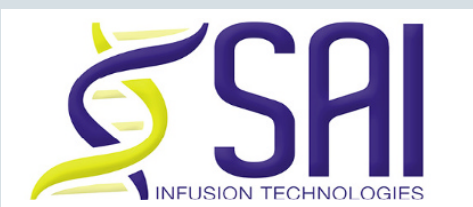

\title{
Tools and techniques for maintaining catheter patency
}

\author{
Candace Rohde-Johnson
}

Indwelling catheters are essential to a large part of animal research, but the use of this equipment is not without challenges. All catheters carry the risk of complications, which can range from loss of patency to life-threatening infection. From surgery to catheter access and catheter maintenance, there are a variety of products and practices that can affect animal health and catheter performance. Employing the best practices from the start can promote efficiency with respect to time and materials, as well as an overall reduction in the number of animals needed for research.

\section{Catheter design and surgical considerations}

The successful use of a catheter depends largely on the catheter's design and the aseptic surgery by which it is introduced. When choosing a catheter, researchers and clinicians must carefully consider the catheter's material, the method by which it is secured, its compatibility with the chosen test solution and the geometry of its tip $^{1-4}$. Not all rounded tips are the same, though, and the rounded tip from SAI has a smooth surface with a more gradual slope that causes less damage during insertion (Fig. 1).

Regardless of a catheter's design, no surgery will be successful without practice and careful attention to aseptic technique. Most infections of intravascular devices arise from contamination by skin flora that takes place during implantation ${ }^{5}$. This can be mitigated by the use of barriers and thorough adherence to aseptic technique, and SAI offers an easy and effective tool for maintaining an aseptic field while simultaneously creating a transparent barrier between a rodent subject and its surgeon (Fig. 2).

\section{Catheter access}

It is also essential that researchers and clinicians use proper technique when accessing an implanted catheter, to ensure its successful use going forward. When possible, they should use a port or valvebased system rather than a pin, as pins are small and hard to handle and therefore increase the risk of introducing a pathogen during access. A port or valve helps to keep the contact surfaces clean and can be integrated into a harness or jacket. Neutral pressure valves with a surface that can easily be swabbed (Fig. 3) make cleaning and care easier ${ }^{6}$.

For vascular access ports, proper cleaning and care of the insertion site is paramount, and a port dressing can help assure these goals. Such a protective pad keeps the insertion area free of debris and can reduce complications such as dislodgement, infection and needlestick incidents ${ }^{7}$. SAI is currently working with customers to test these dressings with a variety of species.

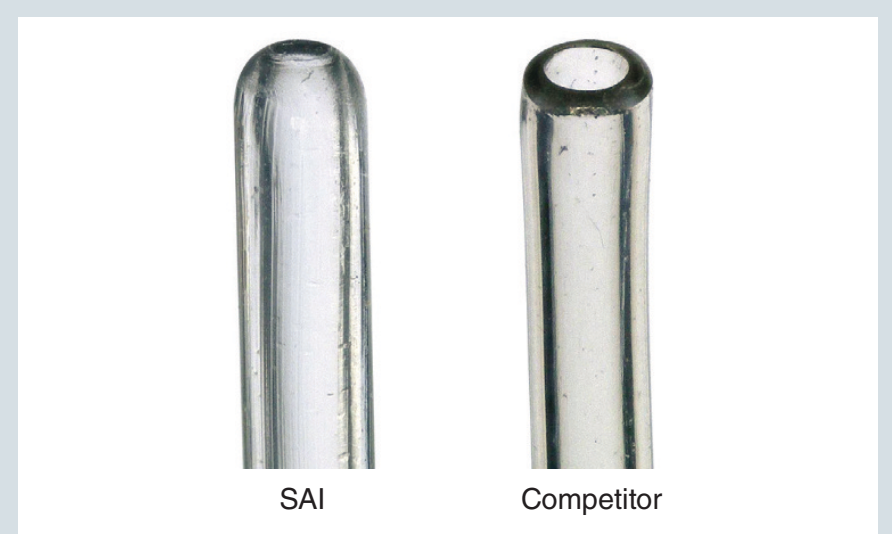

FIGURE 1 | Rounded tips on different catheters. The tip on a SAI catheter (left) is rounded more gradually than those commonly found on competitor products (right).

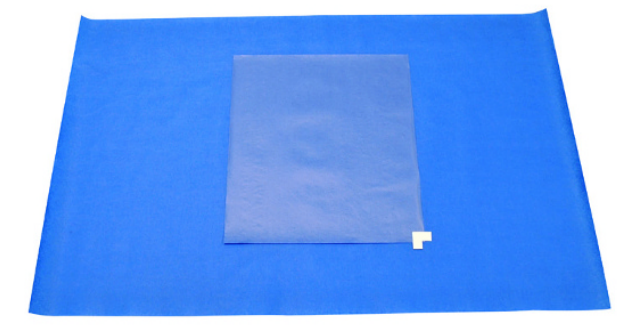

FIGURE 2 | SAI Rodent Surgical Drape with an integrated transparent shield.

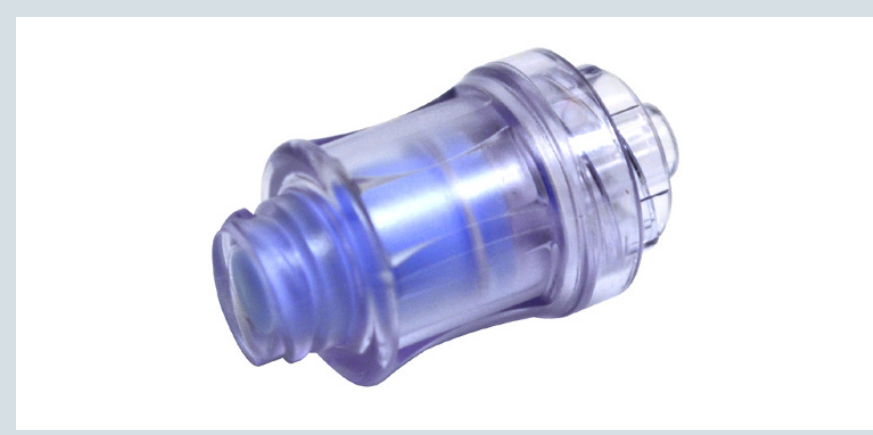

FIGURE 3 | Luer-activated valve that can be easily swabbed for cleaning purposes.

\section{Flushing and locking of catheters}

Following on these steps, it is important to use the right locking solution and flushing routines to maintain a clean catheter ${ }^{8,9}$. One way to manage this challenge while accessing the catheter is to rely on a pre-mixed locking solution. SAI Locking Solutions are 


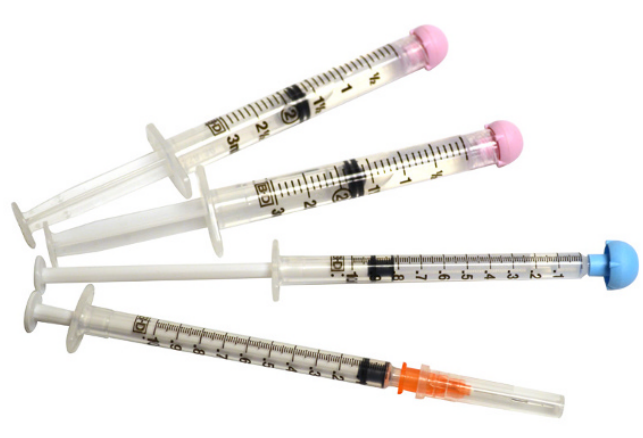

FIGURE 4 | Pre-filled syringes for various applications in preclinical research.

manufactured under clean-room conditions, using pharmaceutical-grade ingredients. These controlled conditions ensure that solutions are clean and batches are identical.

Researchers and clinicians must also consider the tools that are used to deliver these solutions. Prefilled syringes are a well-known time saver in animal research and have produced lower infection rates in clinical studies compared to manually filled syringes ${ }^{10}$. Prefilled syringes are available in a variety of sizes, with a variety of fill solutions and needles, including plastic safety needles. These can be customized to provide appropriate draw, flush and lock syringes for each animal and study (Fig. 4).

\section{Conclusions}

Catheter care involves a variety of important practices and components, beginning with catheter procurement and including the surgical suite, animal care staff and more. With all of these factors, it is easy to see why things might not always go right. Researchers and clinicians can minimize mistakes and increase catheter longevity by keeping the proper tools on hand. Whether the aim is to prevent accidents, save money or reduce the number of animals being used, the right tools can help researchers and clinicians achieve their goals for efficiency and animal well-being.

1. Sherertz, R.J. et al. Contribution of vascular catheter material to the pathogenesis of infection: the enhanced risk of silicone in vivo. J. Biomed. Mater. Res. 29, 635-645 (1995).

2. Weber, K. et al. Pathology in continuous infusion studies in rodents and nonrodents and ITO (infusion technology organisation)-recommended protocol for tissue sampling and terminology for procedure-related lesions. J. Toxicol. Pathol. 24, 113-124 (2011).

3. Swindle, M.M. et al. Vascular acces port (VAP) usage in large animal species. Contemp. Top. Lab. Anim. Sci. 44, 7-17 (2005).

4. O'Farrell, L. Optimal central venous catheter design for longterm blood sampling - a thesis in laboratory animal medicine. Thesis. (Hershey Medical Center, Hershey, PA, 1995).

5. Gandelman, G. et al. Intravascular device infections: epidemiology, diagnosis, and management. Cardiol. Rev. 15, 13-23 (2007).

6. Macklin, D. \& Chernecky, C. Improving IV catheter care to safeguard against deadly infections. Patient Safety \& Quality Healthcare (July/August 2010).

7. Yamamoto, A.J. et al. Sutureless securement device reduces complications of peripherally inserted central venous catheters. J. Vasc. Interv. Radiol, 13, 77-81 (2002).

8. Walter, K., Luo, Y.S., Luo, Y.L., Fisher, T.F. \& Mulder, G.B. Evaluation of three catheter maintenance schedules on patency of jugular vein catheters in CD rats. 2008. Poster, AALAS National Meeting, Indianapolis, IN (9-13 November 2008).

9. Luo, Y. et al. Vascular catheter locking solutions in rats: sodium citrate as an alternative to heparin. 2014. Poster, AALAS National Meeting, San Antonio, TX (19-23 October 2014).

10. Bertoglio, S. et al. Pre-filled normal saline syringes to reduce totally implantable venous access device-associated bloodstream infection: a single institution pilot study. J. Hosp. Infect. 84, 85-88 (2013). 